\title{
Comportamento de risco para transtorno alimentar em universitárias de Viçosa-MG
}

\author{
Risk behavior for eating disorders in female university \\ students from Viçosa-MG
}

Jessica Aparecida Silva', Sílvia Oliveira Lopes², Roberta Stofeles Cecon³, Silvia Eloiza Priore ${ }^{4}$

\begin{abstract}
'Mestranda pelo Programa de Pós-Graduação em Ciência da Nutrição da Universidade Federal de Viçosa.

${ }^{2}$ Doutora em Ciência da Nutrição pela Universidade Federal de Viçosa.

${ }^{3}$ Professora Adjunta Nível 2, da Universidade Federal do Vale do São Francisco

${ }^{4}$ Professora Titular do Departamento de Nutrição e Saúde da Universidade Federal de Viçosa
\end{abstract}

E-mail para correspondência: Jessica Aparecida Silva - jessica.aparecida@ufv.br

\begin{abstract}
Resumo
Objetivo: avaliar comportamento alimentar de risco para o desenvolvimento de transtorno alimentar em acadêmicas de uma instituição do município de ViçosaMG. Métodos: estudo transversal realizado com 104 universitárias com idade igual ou superior a 18 anos, matriculadas no primeiro período. Foram coletados dados de idade, área de conhecimento, curso, antropometria e composição corporal e aplicado o Teste de Atitudes Alimentares (EAT-26). Além da realização da análise descritiva dos dados, utilizou-se o Teste Kolmogorov-Smirnov, o Teste U de MannWhitney, o Teste Qui-Quadrado e o Coeficiente de Spearman. Este estudo foi aprovado pelo Comitê de Ética da Universidade Federal de Viçosa e todas as estudantes assinaram o Termo de Consentimento. Resultados: A prevalência de triagem positiva para anorexia nervosa (EAT+) foi de $21,2 \%(n=22)$. Verificou-se que $76 \%(n=79)$ das participantes foram classificadas como eutróficas pelo índice de Massa Corporal (IMC). Houve associação entre o risco cardiometabólico (pela relação cintura-estatura - RCE) e EAT+ $(p=0,023)$. Observou-se que o grupo EAT+ se difere do EAT- para IMC $(p=0,005)$ e perímetro da cintura $(p=0,022)$; e o grupo Nutrição se difere dos outros para IMC $(p=0,010)$ e RCE $(p=0,013)$. Não se obteve diferença para a prevalência de EAT+ nas diferentes áreas. Conclusão: A elevada prevalência de EAT+ observada nas universitárias é fator preocupante para a saúde física e psíquica, necessitando de políticas de saúde para prevenção de transtornos alimentares de forma efetiva.
\end{abstract}

Palavras-chave: Anorexia Nervosa. Fatores de Risco. Estudantes.

\begin{abstract}
Objective: To evaluate risk behavior for the development of eating disorders in female university from Viçosa-MG. Methods: cross-sectional study with 104 women aged 18 or over, enrolled in the first semester. There were collected data from age, knowledge area, course, from anthropometry and from body composition and the Eating Attitudes Test (EAT-26) was applied. There were made descript statistics,
\end{abstract}


the Kolmogorov-Smirnov test, the Mann-Whitney U-test, the Chi-square test and the Spearman's Coefficient. This study was approved by the Ethics Committee of the Federal University of Viçosa and all the participants signed the consent form. Results: The prevalence of positive screening for anorexia nervosa (EAT+) was 21,2\% $(n=22)$. The eutrophic ones were $76 \%(n=79)$ by Body Mass Index (BMI). There was significant association between cardiometabolic risk (by waist-height ratio WHtR $)$ and EAT+ $(p=0,023)$. EAT+ is statistically different of EAT- for $B M I(p=0,005)$ and circumference waist $(p=0,022)$; the Nutrition group is different from the others for BMI $(p=0,010)$ and WHtR $(p=0,013)$. There wasn't difference $(p=0,415)$ for EAT+ prevalence in the different areas. Conclusion: The high prevalence of EAT+ observed is a cause of concern from the point of view of physical and mental health and it needs specific health-care policies for effective prevention of eating disorder.

Keywords: Anorexia Nervosa. Risk Factors. Students.

\section{INTRODUÇÃO}

Os transtornos alimentares (TA) são distúrbios psiquiátricos que envolvem dimensões pessoais, familiares e socioculturais, caracterizando-se por preocupação excessiva com alimento, peso e forma corporal ${ }^{1,2}$. A angústia de separação também pode ser um dos gatilhos para o desenvolvimento de um TA no que se refere a jovens que deixam o lar para se qualificar para a vida profissional, ingressando em uma universidade ${ }^{3}$.

Destacam-se, dentre os TA, a anorexia nervosa (AN) e a bulimia nervosa (BN), sendo que em ambos prevalece o sentimento de estar gordo ${ }^{1,2}$. O diagnóstico de um TA deve ser realizado por médico treinado e capacitado, preferencialmente por um psiquiatra, utilizando critérios definidos pela Organização Mundial de Saúde (OMS), presentes no Código Internacional de Doenças (CID), e pela Associação de Psiquiatria Americana (APA), que constam no Manual Diagnóstico e Estatístico de Transtornos Mentais (DSM-V) 4 .

O comportamento alimentar também é influenciado pela autopercepção corporal conjecturada como uma insatisfação com sua imagem corporal. A percepção distorcida de seu corpo leva a essa insatisfação e, consequentemente, à realização de práticas inadequadas para controle de peso². Nesse sentido, a imagem corporal é a representação mental do próprio corpo, ou seja, um fenômeno social, pois vai se tecendo com a interação entre os sujeitos, envolvendo as informações recebidas e assimiladas pelo indivíduo, tendo a mídia, portanto, influência notável ${ }^{1,5}$. 
O sexo feminino é frequentemente mais acometido por TA, apresentando dez casos no sexo feminino para cada caso no sexo masculino ${ }^{6}$, sendo mais vulnerável às pressões sociais relacionadas aos padrões de beleza e por isso, a maioria das mulheres apresenta insatisfação quanto ao seu corpo, levando à realização de dietas e controle de seu peso ${ }^{7}$.

A AN, se caracteriza por recusa em manter o peso mínimo adequado à idade com restrição alimentar autoimposta ${ }^{2,7,8,9}$. Pode levar a complicações associadas à desnutrição, como desidratação, distúrbios eletrolíticos, hipoglicemia, hipotermia, hipotensão, insuficiência renal, infertilidade, etc. ${ }^{2,9}$.

Nesse cenário, em que as dietas restritivas que visam ao emagrecimento constituem o fator mais frequente dentre as causas dos TA, tem-se questionado se futuros nutricionistas, que são em sua maioria mulheres jovens, podem estar mais vulneráveis ao desenvolvimento de TA em razão do contexto em que estão inseridas ${ }^{10}$.

Outrossim, questiona-se se a pressão exercida em universitárias para se obter um desempenho no curso associada à pressão para se ter um corpo dentro dos padrões estabelecidos pela sociedade em determinadas profissões como a de nutricionista, podem contribuir para o desenvolvimento de $\mathrm{TA}^{1}$ ou mesmo se a preocupação com o próprio corpo e o peso é fator de escolha do curso ${ }^{11}$.

Nesse sentido, é essencial desenvolver intervenções no âmbito da prevenção, identificando os indivíduos em risco de desenvolver TA, uma vez que os transtornos podem ter longa duração, com recaídas, além de os pacientes serem extremamente resistentes ao tratamento acarretando em graves consequências à saúde, bem como alto custo para o sistema de saúde $\mathrm{e}^{1,4,7,9}$.

Desse modo, a importância desta pesquisa se dá na medida em que se deseja contribuir com estudos na área de nutrição e saúde acerca de comportamento alimentar de risco para TA, trazendo sua contribuição acadêmica. Ademais, este estudo se justifica por sua relevância social, isto é, as instituições de ensino superior precisam conhecer seus discentes para promover políticas voltadas à prevenção de TA.

Portanto, o presente estudo objetivou avaliar comportamento alimentar de risco para o desenvolvimento de transtorno alimentar em acadêmicas de uma instituição 
do município de Viçosa ( $M G$ ), bem como avaliar associações e correlações com o IMC, \%GC e risco cardiometabólico.

\section{MÉTODOS}

\section{Sujeitos}

Trata-se de um estudo transversal realizado com estudantes do sexo feminino com idade igual ou superior a 18 anos das áreas de conhecimento de Ciências Biológicas (curso de Nutrição), Humanas, Exatas e Agrárias, matriculadas e cursando o primeiro período de seus respectivos cursos. A amostra foi determinada por meio do cálculo do tamanho amostral ${ }^{12}$, considerando uma prevalência de 50\% (pois não há estudos prévios que identifiquem a prevalência de triagem para TA na população em questão), variação aceitável de $12 \%$ e intervalo de confiança (IC) de 95\%, totalizando 104 universitárias, 26 em cada área, garantindo maior homogeneização da amostra. A escolha das estudantes das áreas de Humanas, Exatas e Agrárias foi realizada sem a identificação do curso específico, somente pelo reconhecimento da área, como $\mathrm{CH}$ (Ciências Humanas, Letras e Artes), CE (Ciências Exatas e Tecnológicas) e CA (Ciências Agrárias). Para tanto, foi solicitada a permissão das áreas de conhecimento e, em seguida, mediante autorização, as estudantes foram convidadas em sala de aula a participarem da pesquisa. Enquanto na CB (Ciências Biológicas e da Saúde) foram selecionadas somente as estudantes de Nutrição, foco deste trabalho por constituir-se como grupo de risco para desenvolvimento de $\mathrm{TA}^{13}$. Os critérios de inclusão da pesquisa foram: ser do sexo feminino, idade igual ou superior a 18 anos, preencher o Termo de Consentimento Livre e Esclarecido (TCLE) e não estar grávida ou amamentando.

\section{Instrumentos}

A coleta de dados foi realizada por estudante treinada no período de maio a junho de 2018, em local reservado (sala de Nutrição disponibilizada para a realização da pesquisa) para garantia da privacidade das acadêmicas. As estudantes eram avaliadas em horário previamente agendado pela própria participante. Foram coletados dados de idade, área de conhecimento e curso por meio de formulário autopreenchível e, dados de antropometria (peso, estatura e perímetro da cintura PC). O peso foi aferido em balança digital eletrônica, com capacidade máxima de 150 kg e sensibilidade de $50 \mathrm{~g}$ e, a estatura com auxílio de estadiômetro portátil com extensão de 2,13m, dividido em centímetros com precisão de 0,1 cm. Utilizou-se fita 
métrica não elástica para realizar a medida do perímetro da cintura, considerando o ponto médio entre a crista ilíaca e a última costela. A partir dessa medida foi realizada a relação cintura-estatura (RCE), utilizando o ponto de corte de $\geq 0,5$ para risco cardiometabólico ${ }^{14}$.

Foi calculado o Índice de Massa Corporal (IMC) a partir da razão do peso (kg) e altura ao quadrado $\left(\mathrm{m}^{2}\right)$ para classificação do estado nutricional segundo a Organização Mundial de Saúde (OMS) ${ }^{15,16}$, sendo reclassificado em excesso de peso e eutrofia para análise. O percentual de gordura corporal (\%GC) foi determinado por bioimpedância bipolar digital (Tanita) segundo o protocolo proposto por Barbosa ${ }^{17}$ e classificado conforme os pontos de corte estabelecidos por Lohman de acordo com o sexo (baixo peso $<15 \%$; eutrofia $\geq 15<$ e $25 \%$; risco de sobrepeso $\geq 25$ e $<30 \%$; sobrepeso $\geq 30)^{18}$. As estudantes foram orientadas quanto ao protocolo para a avaliação da bioimpedância: não estar em período menstrual (pelo menos 7 dias antes ou após a menstruação); jejum de 12 horas; não praticar exercício físico nas 12h últimas horas antecedentes ao exame; não consumir bebida alcóolica nas $48 \mathrm{~h}$ antecedentes ao exame; não consumir diuréticos pelo menos 7 dias antes ao exame; urinar 30 minutos antes da realização do exame; não usar objetos metálicos durante o exame ${ }^{17}$. Para a avaliação de comportamento alimentar de risco para TA foi utilizado o questionário autopreenchível "Teste de Atitudes Alimentares" (EAT-26) validado por Bighetti et al. ${ }^{19,20}$. Esse teste não é utilizado para diagnóstico de TA, mas identifica comportamentos alimentares de risco para TA, ou seja, realiza triagem para TA com questões direcionadas a comportamentos anoréxicos. Uma vez que não foi possível um médico na equipe para realização do diagnóstico por meio de entrevista clínica (padrão-ouro), utilizou-se o questionário supracitado para triagem, validado para a população brasileira. É um questionário composto por 26 questões com seis opções de resposta, que variam de o a 3 pontos, sendo sempre = 3; muitas vezes $=2$; às vezes $=1$; poucas vezes, quase nunca e nunca $=0$; a questão 25 apresenta resposta invertida. As universitárias que apresentaram pontuação maior ou igual a 20 no EAT foram identificadas como grupo de risco para TA (triagem positiva para anorexia nervosa - EAT+) e aquelas com pontuação menor que 20 como triagem negativa para anorexia nervosa (EAT-). O instrumento apresenta boa reprodutibilidade para estudantes universitárias do sexo feminino ${ }^{21}$. 


\section{Análise dos dados e aspectos éticos}

Os dados coletados foram tabulados no Software Microsoft Excel 2010 e analisados no Software Statistical Package for Social Science (SPSS 20), adotando um nível de significância de $\mathrm{p}<0,05$.

Foi realizada análise descritiva dos dados. Utilizou-se o Teste Kolmogorov-Smirnov para verificar a normalidade das variáveis; o Teste U de Mann-Whitney para comparação dos grupos EAT+ e EAT- quanto ao IMC, PC, \%GC e RCE e; o Teste QuiQuadrado para testar associações entre IMC (excesso de peso e eutrofia), \%GC (elevado e normal), RCE (com risco cardiometabólio e sem risco cardiometabólico) e a triagem positiva ou negativa para TA. Além disso, utilizou-se o Teste Exato de Fisher. Para todas as análises considerou-se um nível de significância de 5\%.

No que tange aos aspectos éticos, a pesquisa foi aprovada pelo Comitê de Ética em Pesquisa com Seres Humanos da Universidade Federal de Viçosa (Parecer 2.573.547/2018).

Todas as participantes receberam retorno sobre as condições nutricionais e de saúde, e os casos que apresentaram alguma alteração dietética e/ou do estado nutricional foram encaminhados ao atendimento nutricional disponibilizado na universidade para acompanhamento de acordo com a necessidade.

\section{RESULTADOS}

Participaram da pesquisa 104 estudantes do primeiro período de seus respectivos cursos, sendo 26 de cada área de conhecimento, com idade média de 19,18 $\pm 2,3$ anos. A prevalência de triagem positiva para anorexia nervosa (EAT + ) foi de $21,2 \%(n=22)$, considerada preocupante, sendo a pontuação mínima e máxima no questionário EAT-26 de 2 e 34, respectivamente (mediana=12 pontos). Quanto ao estado nutricional verificou-se que $76 \%(n=79)$ das participantes foram classificadas como eutróficas, 3,8\% (n=4) como baixo peso e 20,2\% (n=21) como excesso de peso.

Na tabela 1 estão representados os dados de antropometria e composição corporal para os grupos EAT+ e EAT-. Verificou-se que o grupo EAT+ se difere do EAT- para IMC ( $p=0,005), P C(p=0,022)$ e RCE $(p=0,016)$, sendo os valores de tais variáveis significativamente maiores no grupo $\mathrm{EAT}+$, ademais apresentou resultados semelhantes quanto ao \%GC. 
Tabela 1 - Mediana, mínimo e máximo das variáveis antropométricas e de composição corporal das universitárias com triagem positiva e negativa para transtorno alimentar, Viçosa-MG, 2018.

\begin{tabular}{lcccc}
\hline Variáveis & EAT+ (n=22) & EAT- $(\mathbf{n = 8 2 )}$ & $\begin{array}{c}\text { Total } \\
\text { (n=104) }\end{array}$ & \multirow{2}{*}{ p1 } \\
\cline { 2 - 4 } & $\begin{array}{c}\text { Mediana } \\
\text { (mín.-máx.) }\end{array}$ & $\begin{array}{c}\text { Mediana } \\
\text { (mín.-máx.) }\end{array}$ & $\begin{array}{c}\text { Mediana } \\
\text { (mín.-máx.) }\end{array}$ & \\
\hline IMC $(\mathrm{kg} / \mathrm{m} 2)$ & $22,35(15,50-28,60)$ & $21,60(15,80-41,70)$ & $22,2(15,50-41,70)$ & 0,005 \\
PC (cm) & $78,75(59,10-90,00)$ & $74,25(56,00-116,00)$ & $75,0(56,0-116,0)$ & 0,022 \\
\%CC & $28,85(13,80-38,50)$ & $27,15(14,30-44,70)$ & $27,50(13,80-44,70)$ & 0,318 \\
RCE & $0,49(0,36-0,57)$ & $0,46(0,33-0,72)$ & $0,50(0,33-0,72)$ & 0,016 \\
\hline
\end{tabular}

TTeste de Mann-Whitney. IMC = Índice de Massa Corporal; PC = perímetro da cintura; \%GC = percentual de gordura corporal; RCE = relação cintura-estatura; EAT+ = triagem positiva para anorexia nervosa; EAT= triagem negativa para anorexia nervosa. Mín. = mínimo; Máx. = máximo.

Tabela 2 - Índice de Massa Corporal, percentual de gordura e relação-cintura-estatura segundo total e Área do conhecimento de universitárias com triagem positiva e negativa para anorexia nervosa, Viçosa-MG, 2018.

\begin{tabular}{|c|c|c|c|c|}
\hline \multirow[t]{2}{*}{ Variáveis } & $\mathrm{EAT}+(n=22)$ & EAT- $(n=82)$ & $\begin{array}{c}\text { Total } \\
(n=104)\end{array}$ & \multirow[t]{2}{*}{ p1 } \\
\hline & n (\%) & n (\%) & n (\%) & \\
\hline \multicolumn{5}{|l|}{ IMC (kg/m2) } \\
\hline Eutrofia & $15(68,2)$ & $68(82,9)$ & $83(79,8)$ & 0,1422 \\
\hline Excesso de peso & $7(31,8)$ & $14(17,1)$ & $21(20,2)$ & \\
\hline \multicolumn{5}{|l|}{$\% G C$} \\
\hline Normal & $7(31,8)$ & $34(41,5)$ & $41(39,4)$ & 0,411 \\
\hline Elevado & $15(68,2)$ & $48(58,5)$ & $63(60,6)$ & \\
\hline \multicolumn{5}{|l|}{ RCE } \\
\hline Sem risco cardiometabólico & $13(59,1)$ & $68(82,9)$ & $81(77,9)$ & 0,0232 \\
\hline Com risco cardiometabólico & $9(40,9)$ & $14(17,1)$ & $23(22,1)$ & \\
\hline \multicolumn{5}{|l|}{ Área do conhecimento } \\
\hline CH (Ciências Humanas) & $5(19,2)$ & $21(80,8)$ & $26(100)$ & 0,415 \\
\hline IMC (kg/m2) & & & & 1,0002 \\
\hline Eutrofia & $4(19)$ & $17(81)$ & $21(100)$ & \\
\hline Excesso de peso & $1(20)$ & $4(80)$ & $5(100)$ & \\
\hline$\% G C$ & & & & 1,0002 \\
\hline Normal & $2(16,7)$ & $10(83,3)$ & $12(100)$ & \\
\hline Elevado & $3(21,4)$ & $11(78,6)$ & $14(100)$ & \\
\hline RCE & & & & 1,0002 \\
\hline Sem risco cardiometabólico & $4(20)$ & $16(80)$ & $20(100)$ & \\
\hline Com risco cardiometabólico & $1(16,7)$ & $5(83,3)$ & $6(100)$ & \\
\hline CA (Ciências Agrárias) & $7(26,9)$ & $19(73,1)$ & $26(100)$ & 0,415 \\
\hline IMC (kg/m2) & & & & 0,2872 \\
\hline Eutrofia & $5(22,7)$ & $17(77,3)$ & $22(100)$ & \\
\hline Excesso de peso & $2(50)$ & $2(50)$ & $4(100)$ & \\
\hline$\% \mathrm{GC}$ & & & & 0,4072 \\
\hline Normal & $4(36,4)$ & $7(63,6)$ & $11(100)$ & \\
\hline Elevado & $3(20)$ & $12(80)$ & $15(100)$ & \\
\hline RCE & & & & 0,0572 \\
\hline Sem risco cardiometabólico & $3(15,8)$ & $16(84,2)$ & 19 (100) & \\
\hline Com risco cardiometabólico & $4(57,1)$ & $3(42,9)$ & 7 (100) & \\
\hline
\end{tabular}


Continuação da tabela 2

\begin{tabular}{|c|c|c|c|c|}
\hline \multirow{2}{*}{ Variáveis } & $E A T+(n=22)$ & EAT- $(n=82)$ & $\begin{array}{c}\text { Total } \\
(n=104)\end{array}$ & \multirow[t]{2}{*}{ pl } \\
\hline & n (\%) & n (\%) & n (\%) & \\
\hline CE (Ciências Exatas) & $4(15,4)$ & $22(84,6)$ & $26(100)$ & 0,415 \\
\hline IMC (kg/m2) & & & & 0,6252 \\
\hline Eutrofia & $2(12,5)$ & $14(87,5)$ & $16(100)$ & \\
\hline Excesso de peso & $2(20)$ & $8(80)$ & $10(100)$ & \\
\hline$\% G C$ & & & & 0,5422 \\
\hline Normal & - & $6(100)$ & $6(100)$ & \\
\hline Elevado & $4(20)$ & $16(80)$ & $20(100)$ & \\
\hline RCE & & & & 0,5632 \\
\hline Sem risco cardiometabólico & $2(11,1)$ & $16(88,9)$ & $18(100)$ & \\
\hline Com risco cardiometabólico & $2(25)$ & $6(75)$ & $8(100)$ & \\
\hline CB (Ciências Biológicas - Nutrição) & $6(23,1)$ & $20(76,9)$ & $26(100)$ & 0,415 \\
\hline IMC (kg/m2) & & & & 0,0462 \\
\hline Eutrofia & $4(16,7)$ & $20(83,3)$ & $24(100)$ & \\
\hline Excesso de peso & $2(100)$ & $O(0)$ & $2(100)$ & \\
\hline$\% G C$ & & & & 0,1702 \\
\hline Normal & $1(8,3)$ & $11(91,7)$ & $12(100)$ & \\
\hline Elevado & $5(35,7)$ & $9(64,3)$ & $14(100)$ & \\
\hline RCE & & & & 0,0462 \\
\hline Sem risco cardiometabólico & $4(16,7)$ & $20(83,3)$ & $24(100)$ & \\
\hline Com risco cardiometabólico & $2(100)$ & $\mathrm{O}(0)$ & $2(100)$ & \\
\hline
\end{tabular}

'Teste Qui-Quadrado; 2Teste Exato de Fisher. IMC = Índice de Massa Corporal; \%GC = percentual de gordura corporal; $\mathrm{RCE}$ = relação cintura-estatura; EAT + = triagem positiva para anorexia nervosa; EAT- = triagem negativa para anorexia nervosa

Houve associação entre o risco cardiometabólico (pela RCE) e EAT+ (p=0,023). Observou-se que 40,9\% (n=9) das estudantes com EAT+ apresentaram risco cardiometabólico (Tabela 2).

A prevalência de EAT+ nas áreas de CH, CA, CE e CB (Nutrição) foi de 19,2\% (n=5), $26,9 \%(n=7), 15,4 \%(n=4)$ e $23,1 \%(n=6)$, respectivamente, contudo não houve diferença $(p=0,415)($ Tabela 2$)$.

Quanto às universitárias de $\mathrm{CB}$ (Nutrição) constatou-se que houve associação entre o estado nutricional (pelo IMC em categorias) e EAT+ $(p=0,046)$, bem como entre o risco cardiometabólico (pela RCE) e EAT+ $(p=0,046)$. Verificou-se que a maioria das universitárias com EAT+ foram classificadas como eutróficas (Tabela 2).

Na tabela 3 estão representados os dados de antropometria e composição corporal para os grupos CB (Nutrição) e outras áreas ( $\mathrm{CH}, \mathrm{CA}, \mathrm{CE}$ ). Observou-se que o grupo CB (Nutrição) se difere das outras áreas para IMC ( $\mathrm{p}=0,010)$ e RCE $(\mathrm{p}=0,013)$, apresentando valores significativamente maiores para IMC e menores para RCE nas outras áreas. Além disso, apresentou semelhança quanto ao PC, \%GC e Escore EAT. 
Tabela 3 - Mediana, mínimo e máximo das variáveis antropométricas e de composição corporal das universitárias da área de Ciências Biológicas e da Saúde (Nutrição) e outras áreas, Viçosa, 2018.

\begin{tabular}{lcccc}
\hline \multirow{2}{*}{ Variáveis } & $\begin{array}{c}\text { CB (Nutrição) } \\
\text { (n=26) }\end{array}$ & $\begin{array}{c}\text { Outras áreas (CH, CA, CE) } \\
(\mathbf{n = 7 8 )}\end{array}$ & $\begin{array}{c}\text { Total } \\
\text { (n=104) }\end{array}$ & p) \\
\cline { 2 - 4 } & Mediana (mín.-máx.) & Mediana (mín.-máx.) & Mediana (mín.-máx.) & \\
\hline IMC (kg/m2) & $21,25(15,80-27,50)$ & $22,80(15,50-41,70)$ & $22,2(15,50-41,70)$ & 0,010 \\
PC (cm) & $73,00(56,00-86,00)$ & $76,00(59,10-116,00)$ & $75,00(56,00-116,00)$ & 0,118 \\
\% GC & $26,05(18,50-36,40)$ & $27,85(13,80-44,70)$ & $27,50(13,80-44,70)$ & 0,407 \\
RCE & $0,50(0,33-0,70)$ & $0,48(0,36-0,72)$ & $0,50(0,33-0,72)$ & 0,013 \\
Escore EAT & $13,50(4,00-29,00)$ & $12,00(2,00-34,00)$ & $12,00(2,00-34,00)$ & 0,415 \\
\hline
\end{tabular}

'Teste de Mann-Whitney. $\mathrm{CB}$ = Ciências Biológicas e da Saúde; $\mathrm{CH}$ = Ciências Humanas, Letras e Artes; $\mathrm{CA}$ = Ciências Agrárias; $\mathrm{CE}=$ Ciências Exatas e Tecnológicas; IMC = Índice de Massa Corporal; PC = perímetro da cintura; \%GC = percentual de gordura corporal; $R C E$ = relação cintura-estatura; EAT+ = triagem positiva para anorexia nervosa; EAT- = triagem negativa para anorexia nervosa. Mín. = mínimo; Máx. = máximo

\section{DISCUSSÃO}

Encontrou-se mais de 1/5 de prevalência de EAT+ (21,2\%; $n=22)$ nas universitárias por meio do EAT-26, ou seja, a cada cinco universitárias uma apresentou triagem positiva para anorexia nervosa. Tal dado assemelha-se ao estudo de Fiates e Salles ${ }^{7}$ com prevalência de 22,2\% realizado com 221 alunas da Universidade Federal de Santa Catarina (UFSC) e, Alvarenga, Scagliusi e Philippi ${ }^{4}$, com EAT+ de 23,7\% a $30,1 \%$ com estudantes do primeiro e segundo ano de cursos da área da saúde nas cinco regiões do país, ambos utilizando o EAT-26. Destarte, este estudo corrobora os estudos supracitados, salientando a alta prevalência de comportamento alimentar de risco para TA em universitárias.

No que concerne ao estado nutricional, a maioria das estudantes foi classificada como eutrófica $(76 \% ; n=79)$, corroborando os estudos de Penz, Bosco e Vieira2 $(75,8 \%)$ e Kirsten, Fratton e Porta $(85,5 \%)^{10}$. Embora a maioria das universitárias tenha apresentado eutrofia, a prevalência de triagem positiva para anorexia nervosa foi expressiva, o que pode estar associada a uma insatisfação e distorção da imagem corporal independente do peso.

A associação entre risco cardiometabólico (pela RCE) e EAT+ revigora a importância de um acompanhamento atencioso, visto que pode refletir complicações advindas de TA, como a alteração do perfil lipídico e consequentemente a alteração da homeostase metabólica22.

Em relação ao grupo CB (Nutrição) verificou-se a prevalência de 23,1\% (n=6) de EAT+, sendo semelhante ao estudo de Kirsten, Fratton e Porta $(24,7 \%)^{10}$ que avaliou 186 alunas do curso de Nutrição de uma faculdade particular do Rio Grande do Sul de diferentes períodos e, de Fiates e Salles ${ }^{7}$ realizado com 221 estudantes do curso de 
nutrição e áreas desvinculadas da saúde da UFSC que apresentou EAT+ de 25,4\% e $18,7 \%$, respectivamente, ambos utilizando o EAT-26.

Apresentou ainda maior prevalência de EAT+ em relação ao estudo de Laus, Moreira e Costa ${ }^{11}(16,6 \%)$ realizado com 127 estudantes do primeiro período de cursos da área da Saúde e de Humanas da Universidade de Ribeirão Preto (UNAERP), Ribeirão Preto (SP) com o EAT-26 e, menor prevalência em relação à pesquisa de Penz, Bosco e Vieira2 (35\%) com 203 alunas de Nutrição do Centro Universitário UNIVATES Lajeado/RS, por meio do mesmo instrumento. Essa variação justifica-se pelos diferentes pontos de corte utilizados para EAT+, podendo ser 20 ou 21.

Dessa forma, diante da elevada prevalência detectada deve-se ter atenção quanto às acadêmicas não apenas de nutrição, mas de todas as áreas de conhecimento, uma vez que não houve diferença entre os grupos avaliados. Ainda assim, há que se considerar que em determinados grupos, como atletas, dançarinos, modelos, dentre outros, o corpo é utilizado como um instrumento para alcançar um objetivo (boa atuação profissional ou alavancar sua autoestima) ${ }^{23}$. Do mesmo modo, as nutricionistas veem em seus corpos a porta de entrada para o seu profissionalismo. Como exemplo, têm-se pacientes que se pautam na forma corporal do nutricionista para selecionar o melhor para lhe atender. O corpo magro acaba tendo foco central na performance deste profissional, gerando uma maior cobrança em relação à sua forma corporal.

Nesse sentido, o culto ao corpo está relacionado à concepção de beleza e poder e, por conseguinte, pode culminar na insatisfação dos indivíduos com o próprio corpo ${ }^{24}$. Apesar de mulheres de tais cursos e profissões pertencerem ao grupo de risco, atualmente percebe-se uma disseminação do corpo magro como forma de sucesso e estilo de vida, portanto, mulheres de outras profissões, que não tem o corpo como visualização de seu trabalho ou profissionalismo também apresentam comportamento de risco elevado. As mulheres se sentem inadequadas, pois não se encaixam em um ideal de imagem, comumente alterada por programas computacionais, gerando um sentimento de culpa por algo inatingível ${ }^{25}$.

Essa desvalorização da imagem corporal feminina que não atenda aos padrões sociais gera angústia e se constitui em uma forma de controle por meio de um discurso baseado na perfeição. Corpos perfeitos tornam-se, assim, objetivo de vida de mulheres, em um discurso em que se emaranha saúde e beleza. Com esse discurso de beleza disfarçado de saúde o simples ato de comer tem virado uma preocupação 
ou até mesmo uma perturbação em que a inadequação corporal se tornou motivo de constrangimento ${ }^{25}$.

Segundo Garcia, Castro e Soares ${ }^{1}$, prevalências de EAT+ maiores que 20\%, como encontrado nas universitárias e especificamente nos grupos CA (26,9\%;n=7), e CB - Nutrição $(23,1 \%$; n=6), são muito preocupantes. Embora se tenha obtido maior prevalência de EAT+ nas universitárias de CA em relação às de CB (Nutrição), indo de encontro com estudos que apontam a maior prevalência em estudantes de nutrição, deve-se considerar que são escassos os estudos que avaliaram estudantes da área de agrárias em relação à saúde. Geralmente, os estudos avaliam EAT+ entre estudantes da área da saúde ou entre a área de humanas e saúde.

Ademais, não foi esclarecido se o ambiente em que essas estudantes estão imersas influenciaria o desenvolvimento de TA ou se essas pessoas por já serem predispostas buscariam por esta profissão $0^{10}$. Portanto, a diferença de prevalência de EAT+ nos grupos CB (Nutrição) e CA pode estar associada ao fato de as estudantes de nutrição nos períodos finais do curso poder apresentar maior prevalência de comportamentos alimentares de risco para TA devido à influência do ambiente, justificando a menor prevalência do primeiro grupo em relação ao segundo, já que o estudo foi realizado com as discentes do primeiro período do curso. Para tanto, seria necessária a realização de pesquisas em diferentes etapas dos cursos.

Assim, têm-se as duas possibilidades como fatores contribuintes para o desenvolvimento de TA, tanto a suscetibilidade por preocupação excessiva com sua forma corporal quanto ao meio influenciador em que estão inseridas ${ }^{10}$.

A associação entre IMC e EAT+ nas estudantes de nutrição podem indicar a preocupação excessiva com o peso e insatisfação da imagem corporal, dado que a maioria é eutrófica² .

Nesse sentido, segundo Laus, Moreira e $\operatorname{Costa}^{11}$, embora a pressão para emagrecer em determinadas profissões não seja a causa principal de TA, pode contribuir para o desenvolvimento desses. Desse modo, são fundamentais pesquisas que verifiquem comportamentos alimentares de risco para TA para realização de ações preventivas nesses grupos.

Embora as adolescentes constituam um público prevalente de TA, o presente estudo apresentou como limitações a amostragem não probabilística, bem como o tamanho amostral devido ao número de vagas destinadas às áreas de conhecimento avaliadas. 
A avaliação de estudantes no início do curso justifica-se pela maior probabilidade de TA nesse grupo de adolescentes/adultas jovens, mas pode não refletir a influência do ambiente.

\section{CONCLUSÃO}

A preocupação excessiva com o peso e com os alimentos desencadeia um ambiente estressante e uma relação doentia com o corpo, tornando-se fator preocupante para a saúde física e psíquica uma vez observada a elevada prevalência de EAT+ nas universitárias. As associações (risco cardiometabólico e EAT+; IMC e EAT+) encontradas demonstram a importância de um acompanhamento.

Nesse sentido, esta pesquisa apresentou como benefícios o delineamento do perfil das universitárias de nutrição em relação ao seu comportamento alimentar; contribuição com estudos mais abrangentes na área de nutrição e saúde sobre comportamentos alimentares de risco para o desenvolvimento de TA; conhecimento por parte da instituição de ensino de seu alunado para propiciar políticas de saúde com o intuito de garantir a prevenção de TA de forma efetiva.

Conclui-se que o comportamento alimentar de risco para TA é frequente em universitárias e que as considerações realizadas avigoram a necessidade de estudos que aprofundem no conhecimento das causas associadas aos TA em estudantes de nutrição, bem como as consequências para sua vida profissional, uma vez que este grupo não apresentou diferença significativa em relação às outras áreas, podendo demonstrar uma pressão social em relação às mulheres de um modo geral.

\section{REFERÊNCIAS}

1. Garcia CA, Castro TG, Soares RM. Comportamento alimentar e imagem corporal entre estudantes de Nutrição de uma universidade pública de Porto Alegre-RS. Clin. Biomed. Res. 2010; 30(3).

2. Penz LR, Bosco SM, Vieira JM. Risco para desenvolvimento de transtornos alimentares em estudantes de Nutrição, Scientia Med. 2008; 18(3): 124-8.

3. Bucaretchi HA. Anorexia e bulimia nervosa: uma visão multidisciplinar. São Paulo: Casa do Psicólogo; 2003.

4. Alvarenga MS, Scagliusi FB, Philippi ST. Comportamento de risco para transtorno alimentar em universitárias brasileiras. Rev. psiquiatr. clín. 2011; 38(1): 3-7.

5. Garcia LA, Milagres OG, Mourão L, Assis M, Palma A. Auto-percepção da imagem corporal em estudantes de Educação Física e Medicina. Revista Brasileira de Atividade Física \& Saúde. 2011; 16(1): 25-30. 
6. American Psychiatric Association (APA). Manual diagnóstico e estatístico de transtornos mentais: DSM-5. 5. ed. Porto Alegre: Artmed; 2014.

7. Fiates GMR, Salles RK. Fatores de risco para o desenvolvimento de distúrbios alimentares: um estudo em universitárias. Rev. Nutr. Campinas. 2001; 14(3): 3-6.

8. Alves E, Vasconcelos FAG, Calvo MCM, Neves J. Prevalência de sintomas de anorexia nervosa e insatisfação com a imagem corporal em adolescentes do sexo feminino do Município de Florianópolis, Santa Catarina, Brasil. Cad. Saúde Pública. 2008; 24(3): 503-12.

9. Pires R, Pinto P, Santos G, Santos S, Zraik H, Torres L, et al. Rastreamento da frequência de comportamentos sugestivos de transtornos alimentares na Universidade Positivo. Revista de Medicina. 2010; 89(2): 115-23.

10. Kirsten VR, Fratton F, Porta NB. D. Transtornos alimentares em alunas de nutrição do Rio Grande do Sul. Rev Nutr., Campinas. 2009; 22(2): 219-27.

11. Laus MF, Moreira RCM, Costa TMB. Diferenças na percepção da imagem corporal, no comportamento alimentar e no estado nutricional de universitárias das áreas de saúde e humanas. Rev. psiquiatr. Rio Gd. Sul. 2009; 31(3): 192-6.

12. Miot HA. Tamanho da amostra em estudos clínicos e experimentais. J. Vasc. Bras. $2011 ; 10(4): 275-8$.

13. Reinstein N, Koszewski WM, Chamberlin B, Smith-Johnson C. Prevalence of eating disorders among dietetics students: does nutrition education make a difference? J Am Diet Assoc. 1992; 92(8): 949-53.

14. Abeso. Associação Brasileira para o Estudo da Obesidade e da Síndrome Metabólica. Diretrizes Brasileiras de Obesidade (2016). 4.ed. São Paulo: ABESO; 2016.

15. World Health Organization (WHO). Growth reference data for 5-19 years Genebra: WHO; 2007.

16. World Health Organization (WHO). Physical Status: the use and interpretation of anthropometry. Genebra: WHO; 1995.

17. Barbosa KBF. Consumo alimentar e marcadores de risco para a síndrome metabólica em adolescentes do sexo feminino: comparação entre instrumentos de inquérito dietético [dissertação]. Viçosa, MG: Universidade Federal de Viçosa; 2006.

18. Lohman T. Advances in body composition assessment: current issues in exercise science. Human Kinects; 1992. 57-63.

19. Bighetti F, Santos CB, Santos JE, Ribeiro RPP. Tradução e validação do Eating Attitudes Test em adolescentes do sexo feminino de Ribeirão Preto, São Paulo. J. bras. psiquiatr. 2004; 53(6): 339-46.

20. Bighetti F. Tradução e validação do Eating Attitudes Test em adolescentes do sexo feminino na cidade de Ribeirão Preto-SP. 2003. Ribeirão Preto: Universidade de São Paulo, 2003.

21. Magalhães VC, Mendonça GAS. Transtornos alimentares em universitárias: estudo de confiabilidade da versão brasileira de questionários autopreenchíveis. Rev. bras. epidemiol., São Paulo. 2005; 8(3): 236-45. 
22. Cecon, RE. Fatores de risco para doenças cardiovasculares em adolescentes com triagem positiva para transtornos alimentares, de Viçosa - MG. Viçosa:

Universidade Federal de Viçosa - UFV, 2011.

23. Cunha, LCN. Anorexia, bulimia e compulsão alimentar. São Paulo: Atheneu; 2008.

24. Bosi MLM, Luiz RR, Morgado CMC, Costa MLD, Carvalho RJ. Autopercepção da imagem corporal entre estudantes de nutrição: um estudo no município do Rio de Janeiro. J. bras. psquiatr., Rio de Janeiro. 2006; 55(2): 108-13.

25. Alvarenga M, Figueiredo M, Timerman F, Antonaccio C. Nutrição Comportamental. Barueri, SP: Manole; 2015.

Submissão: 23/01/2019

Aprovação: 11/04/2021 